\title{
Gestión educativa docente en la calidad de enseñanza universitaria del programa de contabilidad 2020
}

\author{
Santiago Luis Vela Del Aguila \\ svelape@gmail.com \\ Escuela de posgrado \\ Universidad César Vallejo \\ ORCID: 0000-0001-6874-6528
}

José Manuel Delgado Bardales

jmdelgadob@ucvvirtual.edu.pe

Escuela de posgrado

Universidad César Vallejo

ORCID:0000-0001-6574-2759

Scopus autor ID: 24070333700

Código Renacyt: P0050554

\section{RESUMEN}

La investigación tuvo como objetivo caracterizar la gestión educativa docente en la calidad de enseñanza universitaria del programa de contabilidad 2020. El estudio fue básico de diseño descriptivo con revisión sistemática, las revisiones sistemáticas son investigaciones científicas en las cuales la unidad de análisis son los estudios originales primarios, constituyen una herramienta esencial para sintetizar la información científica disponible. Conclusiones, el 80\% de los estudios relacionados con la gestión educativa influyen en la calidad de enseñanza universitaria, debido a que la gestión educativa docentes demandan políticas, condiciones y consecuencias de las acciones desarrolladas por los gestores y docentes; se puede establecer que la calidad de educación universitaria tiene un impacto social distinto en los diferentes países del mundo, porque la educación es base para el desarrollo de un país. Además, es importante porque el ejercicio profesional depende de ello, de que establezca una adecuada formación basada en competencias para que respondan a las necesidades de los grupos de interés, de la población y sociedad en su conjunto.

Palabras clave: gestión educativa, calidad de enseñanza, universidad. 


\title{
Teaching educational management in the quality of university teaching of the
} accounting program 2020

\begin{abstract}
:
The objective of the research was to characterize the educational management of teachers in the quality of university teaching of the accounting program 2020 . The study was basic of descriptive design with systematic review, systematic reviews are scientific investigations in which the unit of analysis are original studies primary schools, constitute an essential tool for synthesizing the available scientific information. Conclusions, $80 \%$ of the studies related to educational management influence the quality of university teaching, because the educational management of teachers demand policies, conditions and consequences of the actions developed by managers and teachers; It can be established that the quality of university education has a different social impact in the different countries of the world, because education is the basis for the development of a country. In addition, it is important because professional practice depends on it, on establishing adequate training based on competencies so that they respond to the needs of interest groups, the population and society as a whole.
\end{abstract}

Keywords: educational management, teaching quality, university.

Artículo recibido: 03 nov. 2020 Aceptado para publicación: 07 dic. 2020

Correspondencia: svelape@ gmail.com, Conflictos de Interés: Ninguna que declarar 


\section{INTRODUCCIÓN}

La calidad de la enseñanza universitaria en Norteamérica muestra que el interés público por la calidad de la educación tiene una milla de ancho y una pulgada de hondo. La enseñanza universitaria no está sincronizada con la orientación general de la sociedad, son conscientes de que los cambios de política educativa tienen que pasar por un proceso complicado hasta que se hagan realidad. Así mismo, no ha de olvidarse que hasta hace poco el movimiento por la calidad de la educación se identificaba con la filosofía de la competitividad, individualismo y elitismo (perspectiva de la excelencia). Sus partidarios estaban más interesados por el rendimiento de los alumnos con mayores potencialidades que por los otros, y aceptaban el hecho de que la búsqueda de la calidad no beneficiara a los que aprenden más lentamente y con dificultades. Hemos de reconocer que, en la última década, y por factores externos a la universidad, el tema de la calidad ha cobrado especial relevancia: era necesario evaluar en qué medida era posible reducir ciertos costes universitarios dada su escasa contribución a la calidad de la educación universitaria. Inicialmente el motivo no ha sido una loable preocupación por la calidad, sino la presión ejercida por la reducción presupuestaria. Así, las universidades europeas se han tenido que enfrentar con una situación relativamente nueva: la evaluación institucional (Rodríguez,2000, p.2-3)

Además, las universidades peruanas enfrentan una problemática, más grave, evidentemente, es el del bajo nivel académico (p. 40). En gran parte de las facultades los profesores están poco preparados y desmotivados, lo que se traduce en niveles bajos de competencia en el estudiante y egresados. Existe poca investigación de parte de los docentes, siendo ésta de pobre calidad, toda vez que los recursos económicos que las universidades destinan a la labor de investigación de los docentes son ínfimos y, sobre todo, cada vez es más notoria la falta de capacitación. Finalmente, la desconexión entre la universidad y la empresa, afecta tanto la posibilidad de los graduados de insertarse rápidamente en el mercado laboral, como la necesidad del sector empresarial de contar con cuadros que se adapten prontamente a la labor productiva (Mabres, 1994).

La Universidad Nacional Autónoma de Alto Amazonas, se ubica en el distrito de Yurimaguas, provincia de Alto Amazonas, región Loreto. Sus autoridades están conformadas con Comisión Organizadora, en la actualidad se encuentra licenciada por seis años. Asimismo, la presente tesis de investigación ha sido motivada debido a que la Universidad Nacional Autónoma de Alto Amazonas es una casa de estudios nueva que recién ha empezado a impartir enseñanza académica, y actualmente se viene evidenciando la deserción de alumnos y una cantidad considerable de alumnos desaprobados en los cursos del programa de contabilidad. Esto se debe a falta de la mejora en la calidad de la enseñanza por parte de los docentes universitarios. En su PIA-20182019 existe programación sobre capacitación a docentes, sin embargo, a la fecha no se ejecuta capacitación alguna. El presupuesto asignado para investigación es ínfimo. Por todo esto, urge la necesidad de diseñar un modelo de gestión educativa docente para mejorar la calidad de la 
enseñanza universitaria. Por lo mencionado, la investigación se planteó como problema: ¿cómo será la gestión educativa docente en la calidad de enseñanza universitaria del programa de contabilidad 2020? Y como Objetivo: Caracterizar la gestión educativa docente en la calidad de enseñanza universitaria del programa de contabilidad 2020.

\section{ESTRATEGIAS METODOLÓGICAS O MATERIALES Y MÉTODOS}

El tipo de investigación es básica de diseño descriptivo con revisión sistemática, las revisiones sistemáticas son investigaciones científicas en las cuales la unidad de análisis son los estudios originales primarios, constituyen una herramienta esencial para sintetizar la información científica disponible, incrementar la validez de las conclusiones de estudios individuales e identificar áreas de incertidumbre donde sea necesario realizar investigación (Hernández, 2016, p.217). Es descriptiva, porque se recogió información relacionada la gestión educativa docente en la calidad de enseñanza universitaria del programa de contabilidad (Tamayo y Tamayo, 2009, p.54). Por lo mencionado, se revisó información científica y se visualizó como solucionar una necesidad en la organización de la entidad pública, para mejorar la gestión educativa docente en la calidad de enseñanza universitaria del programa de contabilidad y otras deficiencias existentes. La recolección de datos se realizó a través de una tabla de registro de datos y la fuente fue revisión bibliográfica de artículos de investigaciones tanto nacionales como internacionales que tuvieron como tema principal la gestión educativa docente en la calidad de enseñanza universitaria del programa de contabilidad; de todos los artículos que se encontraron, se incluyeron los estudios (10) más importantes según nivel de evidencia y se excluyeron los menos relevantes. Se estableció la búsqueda siempre y cuando se tuvo acceso al texto completo del artículo científico.

La técnica de análisis se desarrolló a través del análisis de la revisión sistemática evaluando cada uno de los artículos para una comparación de los puntos o características en las cuales concuerda y los puntos en los que existe discrepancia entre artículos nacionales e internacionales. Además, de acuerdo a criterios técnicos pre establecidos, se realizó una evaluación crítica e intensiva de cada artículo, a partir de ello, se determinó la calidad de la evidencia y la fuerza de recomendación para cada artículo. 


\section{RESULTADOS Y DISCUSIÓN}

\section{RESULTADOS:}

Tabla 1: Estudios revisados sobre la auditoría gubernamental en la gestión administrativa - operacional municipal

DATOS DE LA PUBLICACIÓN

\begin{tabular}{|c|c|c|c|c|}
\hline 1. Autor & Año & Nombre de la Investigación & Revista donde se ubica la Publicación & Volumen y Número \\
\hline \multirow{3}{*}{ Barrios C. } & \multirow{3}{*}{2018} & \multirow{3}{*}{$\begin{array}{l}\text { Gestión educativa basada en un sistema de calidad en instituciones de } \\
\text { educación universitaria. }\end{array}$} & Revista Docencia Universitaria. & \multirow{3}{*}{ Vol. 19 Issue 1, p53-73. 21p. } \\
\hline & & & $\begin{array}{l}\text { Colombia } \\
\text { http://search.ebscohost.com/login.aspx? } \\
\text { direct=true\&db=fua\&AN=136823455\& }\end{array}$ & \\
\hline & & & lang $=$ es $\&$ site $=$ eds-live & \\
\hline
\end{tabular}

\section{CONTENIDO DE LA PUBLICACIÓN}

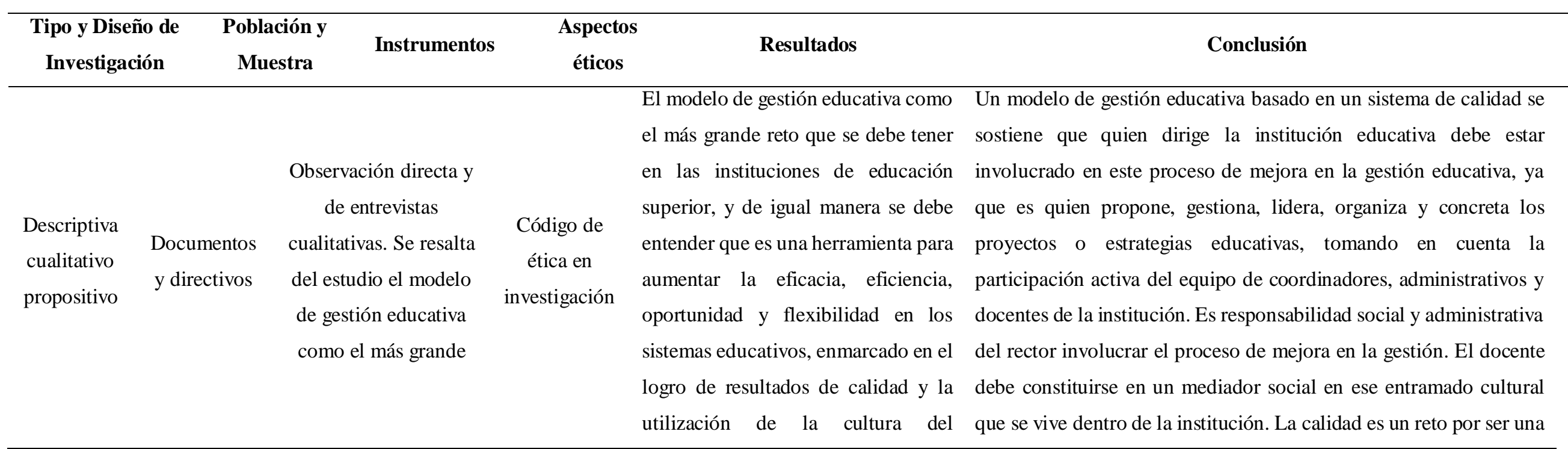

Ciencia Latina Revista Científica Multidisciplinar, Ciudad de México, México. 
seguimiento y la evaluación, como el

instrumento fundamental para

fortalecer la educación, logrando con

ello orientar la toma de decisiones,

las políticas y las planificaciones

hacia los objetivos de la calidad herramienta que aumenta la eficacia, eficiencia, oportunidad y flexibilidad de los sistemas educativos. Es el rector el que debe implementar la cultura evaluativa como herramienta fundamental para el fortalecimiento educacional, procurar como responsabilidad social y ética la buena formación, porque es deber de él facilitar, implementar e incentivar la capacitación permanente.

\begin{tabular}{|c|c|c|c|c|}
\hline 2. Autor & Año & Nombre de la Investigación & Revista donde se ubica la Publicación & Volumen y Número \\
\hline \multirow{3}{*}{$\begin{array}{c}\text { Enríquez, A.,Garofalo, D., \& } \\
\text { Hidalgo, F. }\end{array}$} & \multirow{3}{*}{2018} & & Dilemas Contemporáneos: Educación, Política & \multirow{3}{*}{ Issue, $\mathrm{p} 1-14,14 \mathrm{p}$} \\
\hline & & $\begin{array}{l}\text { La Administración y su contribución en } \\
\text { la Gestión Educativa en las Instituciones } \\
\text { de Educación Superior en el Ecuador. }\end{array}$ & $\begin{array}{l}\text { y Valores. Toluca México. } \\
\text { http://search.ebscohost.com/login.aspx?direct= } \\
\text { true\&db=eue\&AN=130757953\&lang=es\&site }\end{array}$ & \\
\hline & & & =eds-live. & \\
\hline
\end{tabular}

\section{CONTENIDO DE LA PUBLICACIÓN}

\begin{tabular}{|c|c|c|c|c|c|}
\hline $\begin{array}{l}\text { Tipo y Diseño de } \\
\text { Investigación }\end{array}$ & $\begin{array}{c}\text { Población y } \\
\text { Muestra }\end{array}$ & Instrumentos & $\begin{array}{l}\text { Aspectos } \\
\text { éticos }\end{array}$ & Resultados & Conclusión \\
\hline Descriptiva & Artículos & $\begin{array}{c}\text { Ficha de } \\
\text { revisión } \\
\text { documentaria }\end{array}$ & $\begin{array}{c}\text { Código de } \\
\text { ética en } \\
\text { investigación }\end{array}$ & $\begin{array}{l}\text { La idea en el campo de la } \\
\text { administración y la gestión } \\
\text { educativa a nivel superior, es } \\
\text { la calidad de vida y el } \\
\text { progreso de un país } \\
\text { independiente, ambos temas } \\
\text { están ligados a la cobertura, }\end{array}$ & $\begin{array}{l}\text { Esto sin menoscabar la importancia de que operen criterios propios en } \\
\text { el campo de la educación superior. Es decir, la mayor democratización } \\
\text { que debe operarse en el acceso no debe ser leída en términos } \\
\text { antinómicos, sino con la mayor excelencia académica que debe } \\
\text { asegurarse en la educación superior que aporten al cambio en la } \\
\text { estructura productiva, y al mismo tiempo aseguren condiciones de vida } \\
\text { digna y emancipadora. También se deben atacar otras formas de }\end{array}$ \\
\hline
\end{tabular}


calidad y pertinencia de la

formación superior que

brinda a sus ciudadanos $\mathrm{y}$

ciudadanas, y a la inversión

que realiza en ciencia,

tecnología e innovación.

Para el cambio es necesario

que la educación superior se

transforme en un verdadero

mecanismo de movilidad

social ascendente y de

igualdad de oportunidades.

En esta dirección, primero se

debe promover un acceso en

donde

nivel

socioeconómico no afecte.

discriminación negativa, producto de consideraciones de tipo religioso, cultural, étnico, político-partidario, de género, opción sexual, etc. Esto mismo debe observarse no solo en el momento de la incorporación de un estudiante a la educación superior, sino durante todo su tránsito y egreso educativo. A la vez, y sin dejar de ser meritocrático, el sistema de educación superior debe dejar de ser patriarcal. Si bien el acceso y la igualdad de oportunidades en este nivel educativo resulta una de las más importantes deudas sociales a saldar, igual o más empeño hay que poner en la garantía de la excelencia académica, tanto en la enseñanza superior como en la investigación científica.

Ciencia Latina Revista Científica Multidisciplinar, Ciudad de México, México.

ISSN 2707-2207 / ISSN 2707-2215 (en línea), julio-diciembre, 2020, Volumen 4, Número 2.

https://doi.org/10.37811/cl_rcm.v4i2.129 p. 884 


\begin{tabular}{|c|c|c|c|c|}
\hline 3. Autor & Año & Nombre de la Investigación & Revista donde se ubica la Publicación & Volumen y Número \\
\hline Guerra, R., Ramos, F., \& Roque, R. & 2020 & $\begin{array}{l}\text { Aplicación de la norma ISO 21001:2018 } \\
\text { a la calidad de los programas de } \\
\text { posgrado académico. }\end{array}$ & $\begin{array}{l}\text { Revista Cubana de Educación Médica Superior. } \\
\text { Habana Cuba. } \\
\text { http://search.ebscohost.com/login.aspx?direct=true } \\
\& \mathrm{db}=\mathrm{a} 9 \mathrm{~h} \& \mathrm{AN}=142418626 \& \text { lang=es\&site=eds- } \\
\text { live }\end{array}$ & $\begin{array}{l}\text { Vol. } 34 \text { Issue 1, p1-17. } \\
\qquad \text { 17p. } 2 \text { Charts. }\end{array}$ \\
\hline
\end{tabular}

\section{CONTENIDO DE LA PUBLICACIÓN}

\begin{tabular}{|c|c|c|c|c|c|}
\hline Tipo y Diseño de Investigación & $\begin{array}{c}\text { Población y } \\
\text { Muestra }\end{array}$ & Instrumentos & Aspectos éticos & Resultados & Conclusión \\
\hline Cuantitativo pre experimental & $\begin{array}{l}\text { Docentes, } \\
\text { directivos y } \\
\text { estudiantes } \\
\text { de posgrado }\end{array}$ & Pre y postest & $\begin{array}{l}\text { Código de ética } \\
\text { en investigación }\end{array}$ & $\begin{array}{l}\text { La implementación de la norma ISO } 21001 \\
\text { permite ofrecer un mejor servicio educativo, } \\
\text { a partir de evaluar el grado en el que se } \\
\text { satisfacen los requisitos de los estudiantes, } \\
\text { sus familias, los profesores, el mercado } \\
\text { laboral y la sociedad en general. Para ello, el } \\
\text { sistema de gestión de la organización } \\
\text { educativa incluye a todos los procesos, las } \\
\text { personas y las formas de actuación } \\
\text { necesarias para lograr servicios y productos } \\
\text { educativos de calidad. Para demostrar la } \\
\text { aplicación de la norma al posgrado } \\
\text { académico se emplearon como casos de } \\
\text { estudio los programas de maestría del } \\
\text { Centro de Biomateriales. }\end{array}$ & $\begin{array}{l}\text { La norma ISO 21001, aplicada al } \\
\text { posgrado académico, permite } \\
\text { gestionar internamente la calidad de } \\
\text { los servicios formativos para } \\
\text { satisfacer las necesidades y } \\
\text { expectativas de todas las partes } \\
\text { interesadas y lograr categorías } \\
\text { superiores de acreditación }\end{array}$ \\
\hline
\end{tabular}

Ciencia Latina Revista Científica Multidisciplinar, Ciudad de México, México.

ISSN 2707-2207 / ISSN 2707-2215 (en línea), julio-diciembre, 2020, Volumen 4, Número 2. https://doi.org/10.37811/cl rcm.v4i2.129 p. 885 


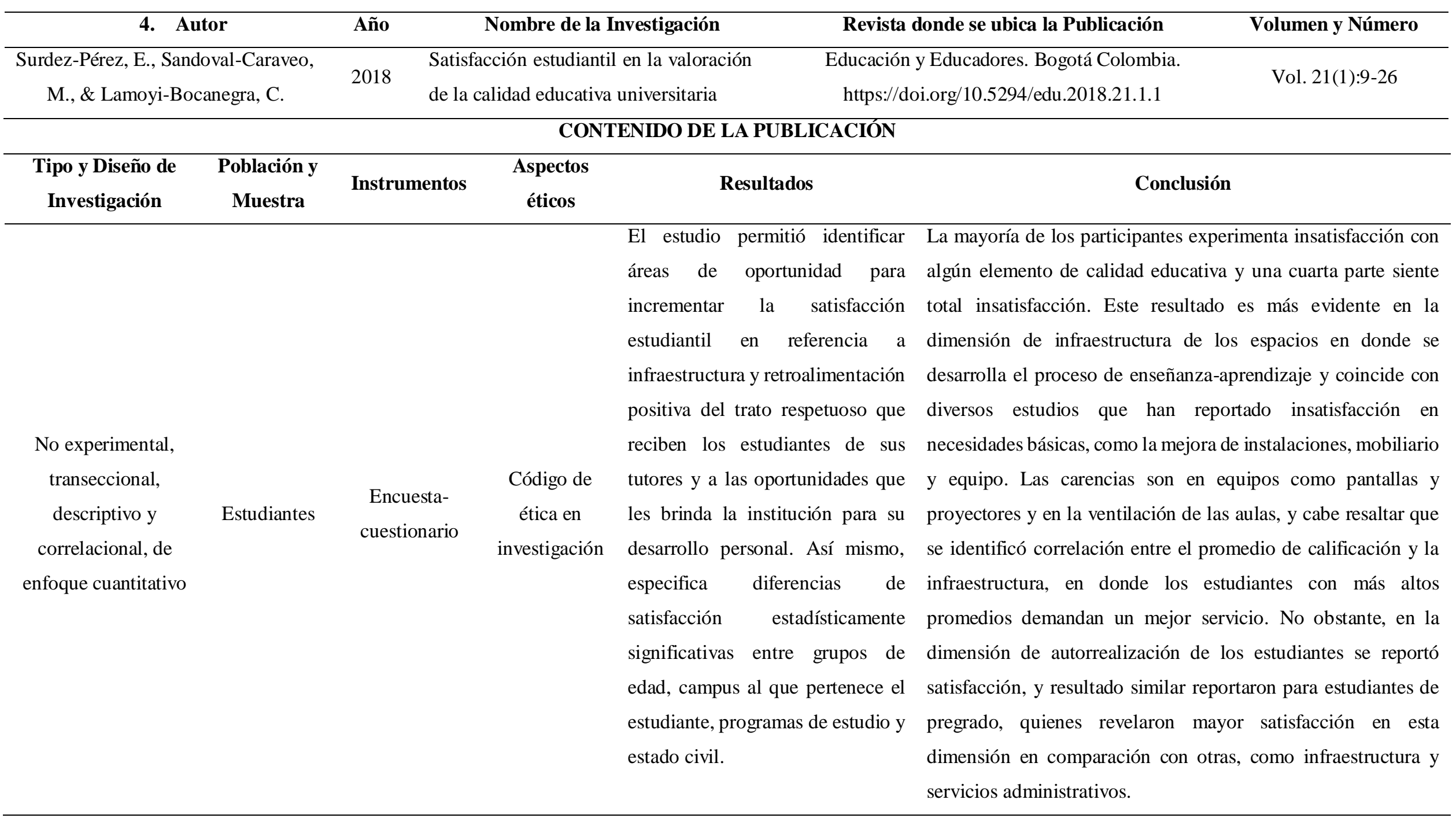

Ciencia Latina Revista Científica Multidisciplinar, Ciudad de México, México. 


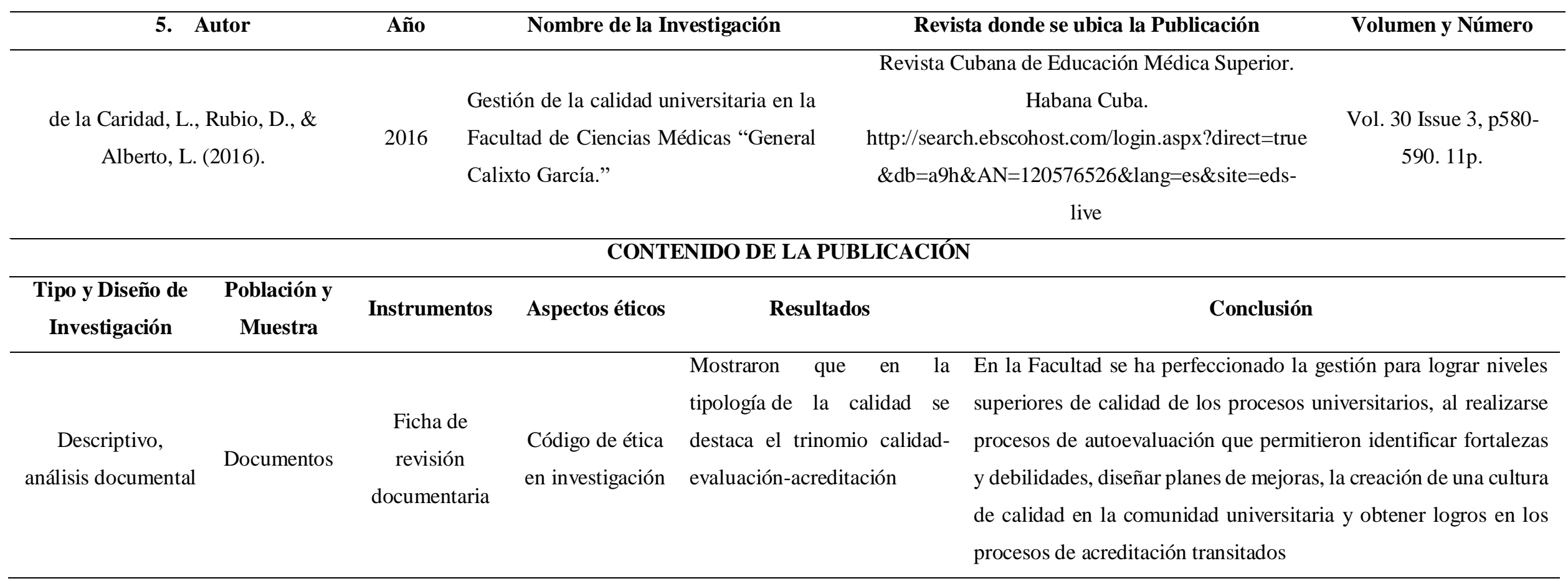


DATOS DE LA PUBLICACIÓN

\begin{tabular}{|c|c|c|c|c|}
\hline 6. Autor & Año & Nombre de la Investigación & Revista donde se ubica la Publicación & Volumen y Número \\
\hline & & & Journal of Alternative Perspectives in the Social & \\
\hline de los Monteros, M. & 2018 & $\begin{array}{l}\text { La Vinculación con la Comunidad Una } \\
\text { Gestión De Calidad En Universidad } \\
\text { Metropolitana. }\end{array}$ & $\begin{array}{l}\text { Sciences. United States of América. } \\
\text {, http://search.ebscohost.com/login.aspx?direct=tru } \\
\text { e\&db=a9h\&AN=133454948\&lang=es\&site=eds- }\end{array}$ & $\begin{array}{l}\text { Vol. } 9 \text { Issue 3, p633-642. } \\
\text { 10p. }\end{array}$ \\
\hline
\end{tabular}

CONTENIDO DE LA PUBLICACIÓN

\begin{tabular}{|c|c|c|c|c|c|}
\hline $\begin{array}{c}\text { Tipo y Diseño de } \\
\text { Investigación }\end{array}$ & $\begin{array}{c}\text { Población y } \\
\text { Muestra }\end{array}$ & Instrumentos & Aspectos éticos & Resultados & Conclusión \\
\hline $\begin{array}{c}\text { Descriptivo, } \\
\text { análisis } \\
\text { documental }\end{array}$ & Documentos & $\begin{array}{c}\text { Ficha de } \\
\text { revisión } \\
\text { documentaria }\end{array}$ & $\begin{array}{l}\text { Código de ética } \\
\text { en investigación }\end{array}$ & $\begin{array}{l}\text { Se cumplió al } 100 \% \text { con la firma de } \\
\text { los convenios interinstitucionales } \\
\text { entre la Universidad Metropolitana } \\
\text { y las escuelas de educación básica, } \\
\text { teniendo una acogida y buena } \\
\text { aceptación en los miembros de la } \\
\text { comunidad educativa. Las escuelas } \\
\text { de educación básica, cuentan con } \\
\text { un conjunto de instrumentos de } \\
\text { diagnóstico, para la determinación } \\
\text { de las potencialidades y } \\
\text { necesidades en la formación de } \\
\text { valores. Se logra un alto nivel de } \\
\text { promoción del proyecto a partir de } \\
\text { la elaboración de los trípticos }\end{array}$ & $\begin{array}{l}\text { La recolección de los datos a partir de los instrumentos aplicados } \\
\text { se cumplió en un } 80 \% \text {, mediante la observación de actividades, } \\
\text { entrevistas a } 1 \text { directivo, a } 9 \text { docentes y las encuestas a } 22 \\
\text { estudiantes, las que evidencian las insuficiencias de la formación } \\
\text { de valores y la necesidad de acciones de capacitación en este } \\
\text { tema. La motivación de los miembros de la comunidad y } \\
\text { estudiantes de la UMET para favorecer la formación de valores } \\
\text { como una perspectiva necesaria para la educación ecuatoriana. Se } \\
\text { cumplió al } 90 \% \text { con la capacitación de las charlas sobre temas de } \\
\text { formación de valores a través de obras literarias, a los padres de } \\
\text { familia quienes asistieron y participaron de manera activa, } \\
\text { superando el porcentaje programado. El } 95 \% \text { de estudiantes del } \\
\text { proyecto participó en la elaboración del material didáctico con el } \\
\text { que dictaron las charlas. Se contó con el } 90 \% \text { de participación en } \\
\text { las charlas sobre situaciones reales de la vida cotidiana que }\end{array}$ \\
\hline
\end{tabular}

Ciencia Latina Revista Científica Multidisciplinar, Ciudad de México, México.

ISSN 2707-2207 / ISSN 2707-2215 (en línea), julio-diciembre, 2020, Volumen 4, Número 2.

https://doi.org/10.37811/cl_rcm.v4i2.129 p. 888 
informativos, campañas, boletines contengan valores, cumpliéndose con lo planteado al inicio del y folletos por parte de los proyecto. Se cumplió con el $80 \%$ de planificaciones y estudiantes en interacción con los capacitaciones reflexivas, se trabajó con los valores de miembros de la comunidad. responsabilidad, solidaridad, honestidad, respeto.

\begin{tabular}{|c|c|c|c|c|}
\hline 7. Autor & Año & Nombre de la Investigación & Revista donde se ubica la Publicación & Volumen y Número \\
\hline $\begin{array}{c}\text { Palomino, I., Calla, K., Yangali, J., } \\
\text { Vásquez, M., Arrátia, G., Luis, J., \& } \\
\text { López, R. }\end{array}$ & 2020 & $\begin{array}{l}\text { Responsabilidad social universitaria y la } \\
\text { calidad de servicio administrativo. }\end{array}$ & $\begin{array}{c}\text { Apuntes Universitarios: Revista de } \\
\text { Investigación, 10(2), 46-63. San Martín Perú. } \\
\text { https://doi.org/10.17162/au.v10i2.440 }\end{array}$ & $\begin{array}{l}\text { Vol. } 10 \text { Issue 2, p46-63, } \\
\qquad 18 \mathrm{p}\end{array}$ \\
\hline
\end{tabular}

\section{CONTENIDO DE LA PUBLICACIÓN}

\begin{tabular}{|c|c|c|c|c|c|}
\hline $\begin{array}{l}\text { Tipo y Diseño de } \\
\text { Investigación }\end{array}$ & $\begin{array}{c}\text { Población y } \\
\text { Muestra }\end{array}$ & Instrumentos & Aspectos éticos & Resultados & Conclusión \\
\hline
\end{tabular}

Investigación

Muestra

Básica, transversal correlacional, no experimental.
De 268 encuestados, el 35,4\% (95) consideran Existe relación significativa entre la variable negativa la responsabilidad social universitaria en la responsabilidad social de la universidad y la universidad, seguido por un 33,2\% (89) quienes la calidad del servicio administrativo en la consideran regular; otro $14,6 \%$ (39) la consideran universidad; esto implica afirmar que: cuanto buena; el 8,6\% (23) la consideran deficiente y por mayor sea la responsabilidad social de la último solo un $8.2 \%$ (22) consideran que es universidad, mayores serán los niveles de eficiente. Si se verifica el promedio $=58,22$ se calidad del servicio

asume que la responsabilidad social universitaria según los encuestados es mala. el 51,9\% (139) considera regular a los impactos organizacionales en la universidad, seguido por un $17,2 \%$ (46) que la consideran buena; otro $16 \%$ (43) la consideran 
mala; un 10,1\% (27) la consideran deficiente y por

último solo un $4,9 \%$ (13) consideran que es

eficiente. Si se verifica el promedio $=15,66$ se

asume que los impactos organizacionales según los

encuestados, es regular

\begin{tabular}{|c|c|c|c|c|}
\hline 8. Autor & Año & Nombre de la Investigación & Revista donde se ubica la Publicación & Volumen y Número \\
\hline & & & Dilemas Contemporáneos: Educación, Política y & \\
\hline $\begin{array}{c}\text { Romero, A., Flores, D., Flores, E., \& } \\
\text { Luzuriaga, M. }\end{array}$ & 2020 & $\begin{array}{l}\text { Gestión de la calidad en instituciones de } \\
\text { educación superior. (Spanish). }\end{array}$ & $\begin{array}{c}\text { Valores. Toluca México } \\
\text { http://search.ebscohost.com/login.aspx?direct=true } \\
\& \mathrm{db}=\text { eue\&AN=141887586\&lang=es\&site=eds- }\end{array}$ & Issue, Vol. 7, p1-15, 15p \\
\hline
\end{tabular}

\section{CONTENIDO DE LA PUBLICACIÓN}

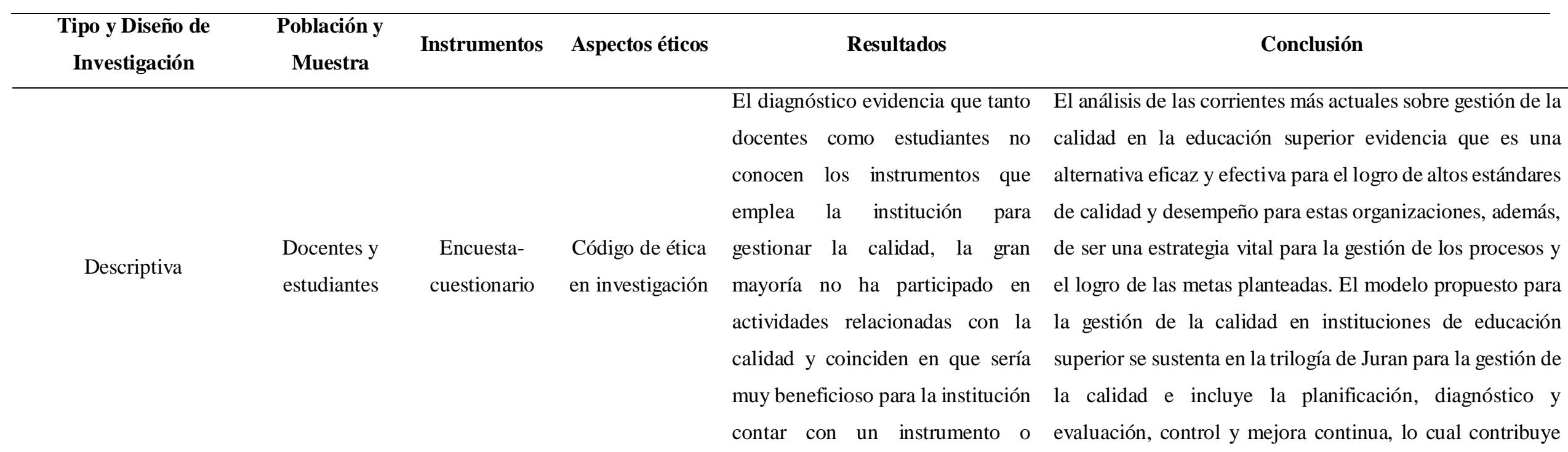

Ciencia Latina Revista Científica Multidisciplinar, Ciudad de México, México.

ISSN 2707-2207 / ISSN 2707-2215 (en línea), julio-diciembre, 2020, Volumen 4, Número 2.

https://doi.org/10.37811/cl_rcm.v4i2.129 p. 890 


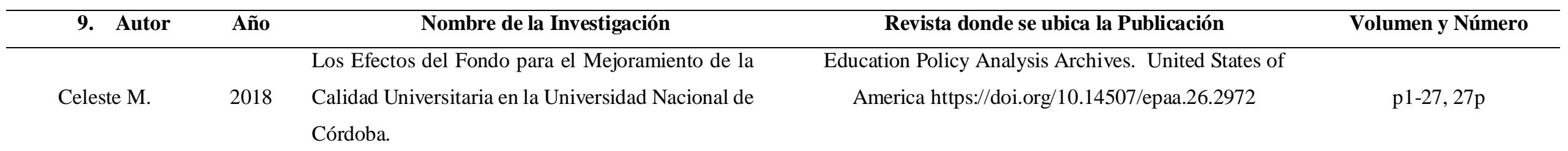

\section{CONTENIDO DE LA PUBLICACIÓN}

\begin{tabular}{|c|c|c|c|c|c|}
\hline $\begin{array}{l}\text { Tipo y Diseño de } \\
\text { Investigación }\end{array}$ & $\begin{array}{c}\text { Población y } \\
\text { Muestra }\end{array}$ & Instrumentos & Aspectos ético & Resultados & Conclusión \\
\hline Descriptiva & $\begin{array}{l}\text { Docentes y } \\
\text { estudiantes }\end{array}$ & $\begin{array}{c}\text { Guía de } \\
\text { entrevista y } \\
\text { documentos } \\
\text { específicos }\end{array}$ & $\begin{array}{c}\text { Código de } \\
\text { ética en } \\
\text { investigación }\end{array}$ & $\begin{array}{l}\text { Desde el esquema de análisis de Ball } \\
\text { (1992, 1994), el cambio en la política de } \\
\text { financiamiento llevada a cabo en } \\
\text { Argentina en los años 90, y en particular } \\
\text { el modo de relacionarse el Estado con } \\
\text { las universidades, no resultó un } \\
\text { acontecimiento aislado. Cabe advertir } \\
\text { que estuvo atravesado por un "Contexto } \\
\text { de Influencia" en términos de Ball, que } \\
\text { en el caso particular de nuestro país } \\
\text { provino principalmente de las } \\
\text { recomendaciones realizadas por las }\end{array}$ & $\begin{array}{l}\text { Las reformas en el sector de la educación superior forman parte } \\
\text { de un contexto más amplio de las reformas del Estado. Desde } \\
\text { los años } 80 \text { en el continente europeo, y desde los años } 90 \text { en el } \\
\text { contexto latinoamericano, comenzaron a implementarse un } \\
\text { conjunto de transformaciones signadas por la desregulación de } \\
\text { los sectores de la economía, un mayor control de los gastos } \\
\text { públicos, hasta medidas anti-inflacionarias en los países en } \\
\text { desarrollo especialmente. Demostrando la presencia de un } \\
\text { Estado neoliberal. En tanto que los patrones de cambio en el } \\
\text { sector universitario estuvieron signados por el reemplazo del } \\
\text { rol del Estado paternalista por un rol observador y de mayor } \\
\text { control del sector. Este nuevo rol fue implementado por } \\
\text { cambios en las políticas de financiamiento hacia las }\end{array}$ \\
\hline
\end{tabular}


agencias internacionales como CEPAL, instituciones, a partir de la diversificación de fuentes

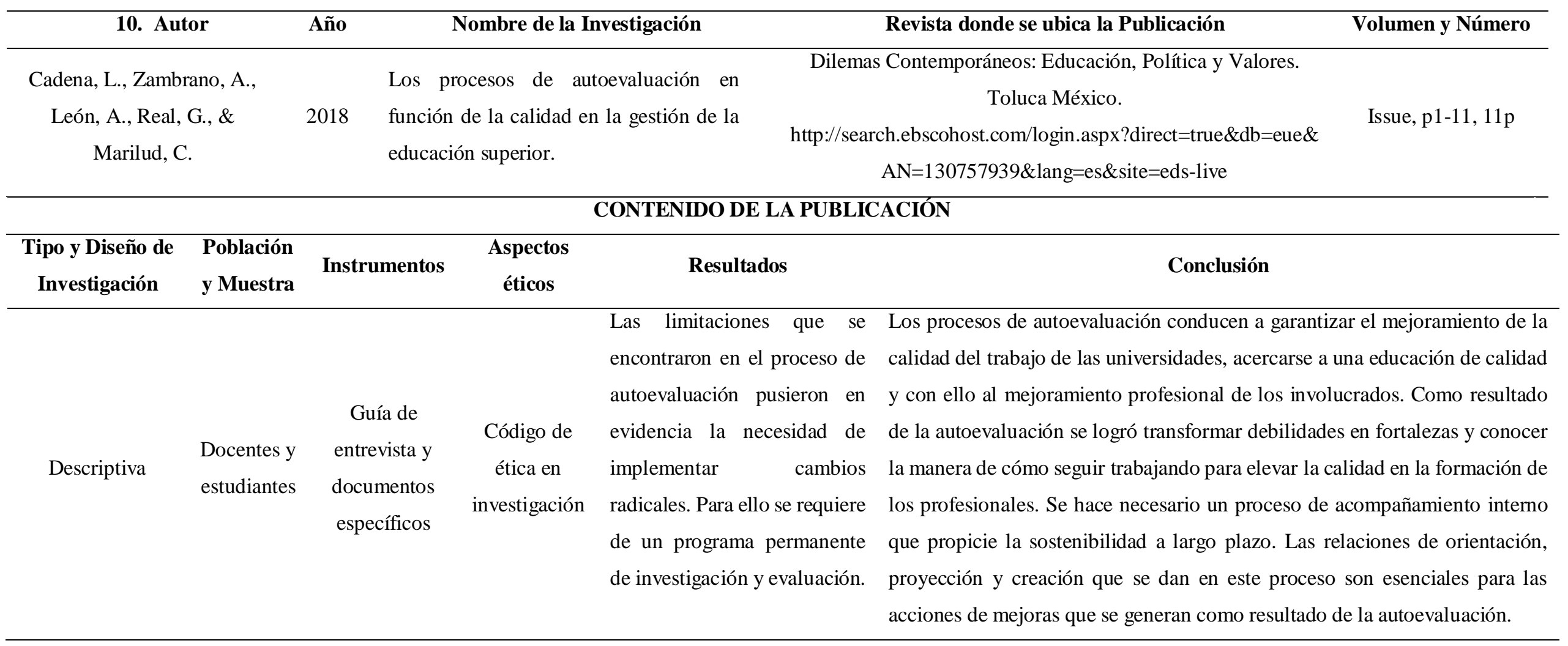

Ciencia Latina Revista Científica Multidisciplinar, Ciudad de México, México. 


\section{DISCUSIÓN}

Posterior a la revisión sistemática de los artículos, el 100\% corresponde a América Latina y el Caribe, a los países Colombia 20\%, México 30\%, Cuba 20\%, EE.UU. 20\% y Perú 10\%. En relación a los diseños y tipos de estudios el 100\% son descriptivos de tipo básica, con las variantes de cualitativo propositivo, análisis documental, cuantitativo pre experimental, correlacional, transversal.

Barrios C., en relación a la gestión educativa docente con la calidad de enseñanza universitaria, concluye que un modelo de gestión educativa es el más grande reto que se debe tener en las instituciones de educación superior, y de igual manera es una herramienta para aumentar la eficacia, eficiencia, oportunidad y flexibilidad en los sistemas educativos, enmarcado en el logro de resultados de calidad y la utilización de la cultura del seguimiento y la evaluación, como el instrumento fundamentales para fortalecer la educación, logrando con ello orientar a la toma de decisiones, las políticas y las planificaciones hacia los objetivos de la calidad.

Enríquez, A., Garofalo, D., \& Hidalgo, F., según la gestión educativa docente con calidad de enseñanza, concluye que la idea en la administración y la gestión educativa a nivel superior, es la calidad de vida y el progreso de un país independiente, ambos temas están ligados a la cobertura, calidad y pertinencia de la formación superior que brinda a sus ciudadanos y ciudadanas, y a la inversión que realiza en ciencia, tecnología e innovación. Para iniciar un cambio es necesario que la educación superior se transforme en un verdadero mecanismo de movilidad social ascendente y de igualación de oportunidades en nuestro país. En esta dirección, primero se debe promover un acceso en donde el nivel socioeconómico no constituya un impedimento para ingresar, ya sea a través de becas, ayudas económicas, políticas de cuotas o créditos educativos.

Sin embargo, Guerra, R., Ramos, F., \& Roque, R., en el marco de la gestión educativa docentes en la calidad de enseñanza universitaria, concluye que, la norma ISO 21001, aplicada al posgrado académico, permite gestionar internamente la calidad de los servicios formativos para satisfacer las necesidades y expectativas de todas las partes interesadas y lograr categorías superiores de acreditación. Ella evalúa de satisfacción de estudiantes, de las familias y profesores, el mercado laboral y sociedad en general.

Surdez-Pérez, E., Sandoval-Caraveo, M., \& Lamoyi-Bocanegra, C., según la gestión educativa en la calidad de enseñanza universitaria determina que, la insatisfacción es evidente en la dimensión de infraestructura de los espacios en donde se desarrolla el proceso de enseñanza-aprendizaje y coincide con diversos estudios que han reportado insatisfacción en necesidades básicas, como la mejora de instalaciones, mobiliario y equipo, donde resalta que se identificó correlación entre el promedio de calificación y la infraestructura, donde los estudiantes con más altos promedios demandan un mejor servicio. No obstante, en la dimensión de autorrealización de los estudiantes se reportó satisfacción, y resultado similar reportaron para estudiantes de pregrado, quienes revelaron mayor satisfacción en 
esta dimensión en comparación con otras, como infraestructura y servicios administrativos. Además, los estudiantes perciben libertad y autonomía para el desempeño de sus actividades académicas.

De la Caridad, L., Rubio, D., \& Alberto, L., según la gestión educativa en la calidad de enseñanza universitaria, establece que la facultad ha perfeccionado la gestión para lograr niveles superiores de calidad de los procesos universitarios, al realizarse procesos de autoevaluación que permitieron identificar fortalezas y debilidades, diseñar planes de mejora, la creación de una cultura de calidad en la comunidad universitaria y obtener logros en los procesos de acreditación transitados permitieron mejores resultados en la enseñanza universitaria.

De los Monteros, M., según la gestión educativa, concluye que evidencian las insuficiencias de la formación de valores y la necesidad de acciones de capacitación, como la motivación de los miembros de la comunidad universitaria para favorecer la formación de valores. Se cumplió la capacitación de las charlas sobre temas de formación de valores a través de obras literarias, a los padres de familia quienes asistieron y participaron de manera activa, superando lo programado, observándose mayor involucramiento de los padres que los estudiantes.

Palomino, I., Calla, K., Yangali, J., Vásquez, M., Arrátia, G., Luis, J., \& López, R., en relación a la calidad educativa universitaria, concluye que existe relación significativa entre la variable responsabilidad social de la universidad y la calidad del servicio administrativo en la universidad; esto implica afirmar que: cuanto mayor sea la responsabilidad social de la universidad, mayores serán los niveles de calidad del servicio, por lo tanto demanda mayor proyección de la universidad a la sociedad.

Romero, A., Flores, D., Flores, E., \& Luzuriaga, M., concluye que, las corrientes más actuales sobre gestión de la calidad en la educación superior evidencia que es una alternativa eficaz y efectiva para el logro de altos estándares de calidad y desempeño para estas organizaciones, además, de ser una estrategia vital para la gestión de los procesos y el logro de las metas planteadas. El modelo de gestión de la calidad en instituciones de educación superior se sustenta en la trilogía de Juran para la gestión de la calidad e incluye la planificación, diagnóstico y evaluación, control y mejora continua, lo cual contribuye significativamente a gestionar la calidad de los procesos que en la institución se desarrollan.

Celeste M., en relación a la gestión educativa docente universitaria, concluye que, está demostrado la presencia de un Estado neoliberal. En tanto que los patrones de cambio en el sector universitario estuvieron asignados por el reemplazo del rol del Estado paternalista por un rol observador y de mayor control del sector. Este nuevo rol fue implementado por cambios en las políticas de financiamiento hacia las instituciones, a partir de la diversificación de fuentes presupuestarias y modos de asignación, asociadas a la consecución de ciertos criterios de calidad, internos y externos, por parte de las instituciones. 
Cadena, L., Zambrano, A., León, A., Real, G., \& Marilud, C., según la calidad de enseñanza universitaria, concluye que, los procesos de autoevaluación conducen a garantizar el mejoramiento de la calidad del trabajo de las universidades, acercarse a una educación de calidad y con ello al mejoramiento profesional de los involucrados. Permitiendo la autoevaluación transformar debilidades en fortalezas y conocer la manera de cómo seguir trabajando para elevar la calidad en la formación de los profesionales. Sin embargo, el acompañamiento interno es importante porque propicia la sostenibilidad a largo plazo. Además, debe existir las relaciones de orientación, proyección y creación en este proceso para las acciones de mejoras que se generan como resultado de la autoevaluación.

\section{CONCLUSIÓN O CONSIDERACIONES FINALES}

La revisión sistemática de los 10 artículos científicos, sobre la gestión educativa docente en la calidad de enseñanza universitaria, corresponden a América latina y el caribe, y a diferentes bases de datos, todos ellos pertenecen al tipo y diseño de estudios descriptivos.

Posterior a la revisión sistémica, se concluye que el $80 \%$ de los estudios relacionados con la gestión educativa influyen en la calidad de enseñanza universitaria, debido a que la gestión educativa docentes demandan políticas, condiciones y consecuencias de las acciones desarrolladas por los gestores y docentes; se puede establecer que la calidad de educación universitaria tiene un impacto social distinto en los diferentes países del mundo, porque la educación es base para el desarrollo de un país.

Por otro lado, el $80 \%$ concluye que la calidad de la enseñanza universitaria es importante porque el ejercicio profesional depende de ello, de que establezca una adecuada formación basada en competencias para que respondan a las necesidades de los grupos de interés, de la población y sociedad en su conjunto. Así mismo las consecuencias de una deficiente gestión educativa docente y de calidad de enseñanza universitaria van en incremento y afectan socialmente al país, a las familias y a las instituciones formadoras porque el egresado no cumple las competencias para el ejercicio profesional y los colegios profesionales se ven en la responsabilidad de actuar como entes formadores para vencer esta deficiencia que es responsabilidad netamente de las universidades.

Por otro lado, existe las condiciones establecidas por norma ISO 21001, aplicada a las diferentes áreas académicas, permite gestionar internamente la calidad de los servicios formativos con resultados aceptables que permiten satisfacer las necesidades y expectativas de todas las partes interesadas bajo un enfoque de calidad.

\section{LISTA DE REFERENCIAS}

Barrios C. (2018). Gestión educativa basada en un sistema de calidad en instituciones de educación universitaria. Revista Docencia Universitaria. Vol. 19 Issue 1, p53-73. 21p. Colombia. Disponible

en: 
http://search.ebscohost.com/login.aspx?direct=true $\& \mathrm{db}=$ fua $\& A N=136823455 \&$ lang=es $\&$ site =eds-live

Cadena, L., Zambrano, A., León, A., Real, G., \& Marilud, C. (2018). Los procesos de autoevaluación en función de la calidad en la gestión de la educación superior. Dilemas Contemporáneos: Educación, Política y Valores. Issue, p1-11, 11p. Toluca México. Disponible en: http://search.ebscohost.com/login.aspx?direct=true \& db=eue\& AN=130757939\&lang=es\&sit e=eds-live

Celeste M. (2018). Los Efectos del Fondo para el Mejoramiento de la Calidad Universitaria en la Universidad Nacional de Córdoba. Education Policy Analysis Archives. p1-27, 27p. United States of America https://doi.org/10.14507/epaa.26.2972

De la Caridad, L., Rubio, D., \& Alberto, L. (2016). Gestión de la calidad universitaria en la Facultad de Ciencias Médicas "General Calixto García." Revista Cubana de Educación Médica Superior. Vol. 30 Issue 3, p580-590. 11p. Habana Cuba. Disponible en: http://search.ebscohost.com/login.aspx?direct=true \&db=a9h\&AN=120576526\&lang=es\&sit $\mathrm{e}=$ eds-live

De los Monteros, M. (2018). La Vinculación con la Comunidad Una Gestión De Calidad En Universidad Metropolitana. Journal of Alternative Perspectives in the Social Sciences. Vol. 9 Issue 3, p633-642. 10p. United States of América. Disponible en: http://search.ebscohost.com/login.aspx?direct=true \&db=a9h\&AN=133454948\&lang=es\&sit e=eds-live

Enríquez, A., Garofalo, D., \& Hidalgo, F. (2018). La Administración y su contribución en la Gestión Educativa en las Instituciones de Educación Superior en el Ecuador. Dilemas Contemporáneos: Educación, Política y Valores. Issue, p1-14, 14p. Toluca México. Disponible

en: http://search.ebscohost.com/login.aspx?direct=true \& db=eue\& $A N=130757953 \& l a n g=e s \&$ sit e=eds-live.

Guerra, R., Ramos, F., \& Roque, R. (2020). Aplicación de la norma ISO 21001:2018 a la calidad de los programas de posgrado académico. Revista Cubana de Educación Médica Superior. Vol. 34 Issue 1, p1-17. 17p. 2 Charts. Habana Cuba. Disponible en: http://search.ebscohost.com/login. aspx?direct=true \&db=a9h\& AN=142418626\&lang=es\&sit e=eds-live

Palomino, I., Calla, K., Yangali, J., Vásquez, M., Arrátia, G., Luis, J., \& López, R. (2020). Responsabilidad social universitaria y la calidad de servicio administrativo. Apuntes Universitarios: Revista de Investigación, 10(2), 46-63. Vol. 10 Issue 2, p46-63, 18p. San Martín Perú. https://doi.org/10.17162/au.v10i2.440 
Rodríguez, S. (2000). Calidad de la enseñanza Universitaria. Artículo científico. Universidad de Barcelona. https://www.researchgate.net/publication/28073791_La_calidad_en_la_Ensenanza_Universit aria

Romero, A., Flores, D., Flores, E., \& Luzuriaga, M. (2020). Gestión de la calidad en instituciones de educación superior. (Spanish). Dilemas Contemporáneos: Educación, Política y Valores. Issue, Vol. 7, p1-15, 15p Toluca México. Disponible en: http://search.ebscohost.com/login.aspx?direct=true \& db=eue\& AN=141887586\&lang=es\&sit e=eds-live

Surdez-Pérez, E., Sandoval-Caraveo, M., \& Lamoyi-Bocanegra, C. (2018). Satisfacción estudiantil en la valoración de la calidad educativa universitaria Educación y Educadores. Vol. 21(1):9-26. Bogotá Colombia. Disponible en: https://doi.org/10.5294/edu.2018.21.1.1 